\title{
Faecal quality control
}

\author{
Faecal microbiota transplantation can efficiently treat recurring Clostridioides difficile infection and is being \\ investigated for other indications. However, strict quality control of the donor stool is necessary to avoid putting \\ patients at unnecessary risk.
}

A brief foray into the online world of 'do-it-yourself' faecal microbiota transplantation (FMT) brings up a wealth of videos and 'recipes'. Touted as the miracle cure for many ailments, it is easy to understand why 'do-it-yourself' approaches are flourishing. Although unsavoury, the recommended process seems simple enough: mix faeces and saline in a kitchen blender, strain this sludge or not (the advice here is mixed), and pour the resulting mush into an enema bottle or gelatin pill capsules for home administration. One can find recommendations for using FMT for all kinds of indications, from the more mainstream treatment of antibiotic-related enteric infections to mood disorders and psoriasis. Its use is even recommended to ameliorate bad breadth, although this seems unlikely to work through oral administration. All jokes aside, this is a worrying trend. The proven success of FMT in treating recurring Clostridioides difficile infection (rCDI), has also led to the proliferation of an unregulated global industry that offers the procedure to desperate patients for other, often not rigorously tested, indications, and often with unclear donor recruitment and stool quality control practices.

Although not mainstream in Western medicine until recently, FMT is far from new. Faecal medicine was described 3,000 years ago in the oldest traditional Chinese medicine prescription book and in the second century, another Chinese medical text described the oral use of a human faecal suspension to treat food poisoning, which is considered the first written record of using human faeces to treat disease. In seventeenth century Europe, the Italian Fabrizio and the German Paullini documented the use of FMT, and the American microbiologist Stan Falkow candidly recalled his role in preparing first-generation 'poop pills' (http://bit.ly/2LEgrSa) to reconstitute the gut communities of surgical patients in 1957. This was a year before Eiseman and colleagues published the first case report of FMT in Western medicine after successfully treating four people suffering from pseudomembranous colitis before C. difficile was the known cause (Surgery 44, $856-859 ; 1959)$. Since then, a growing body of work has consistently shown the efficacy of FMT in treating rCDI, but the approach did not become a household concept until the early 2000s when C. difficile prevalence and mortality rates started rising in North America and Europe. Western medical professional societies have advocated for FMT as standard of care for rCDI and the regulatory agencies of most Western countries allow clinicians to provide FMT for this indication, despite considering it an experimental therapy. Research is ongoing to assess the therapeutic value of FMT for various other conditions, including enteric infection with other multidrug-resistant bacteria, inflammatory bowel disease, ulcerative colitis, graft-versus-host disease, metabolic disease or autism. However, its use for these indications is only allowed within a clinical trial.

The most obvious risk of FMT, although not the only one, is the possibility of transmitting infectious agents, which underscores the importance of rigorously vetting the health of the donor and screening of the stool before transplantation. This is something that does not usually occur in an unregulated FMT context and, perhaps surprisingly, does not always occur in more regulated scenarios either. The latter became apparent a few weeks ago when the FDA reported that one immunosuppressed patient had died and another suffered serious complications after receiving investigational FMT from the same donor, whose stool was later found to contain multidrug-resistant Escherichia coli. As a consequence, the FDA has determined that certain donor and stool controls will be necessary for any future investigational use of FMT and has halted several clinical trials until such measures are put in place.

Strict quality control and screening should be an essential part of the FMT procedure, as an increase in serious adverse events in the context of investigational
FMT could lead to harder regulations and make access more difficult for patients, which would likely increase the demand for unregulated or 'at-home' FMT. However, obtaining safe stool for faecal transplantation is not trivial. In the US, a not-for-profit stool bank, OpenBiome (http://bit.ly/30nsE1B), was founded in 2012 and now supplies stool for most of the US investigational FMT procedures. Stool donors are selected through strict health screens and are closely monitored; additionally, samples are sequenced to screen for pathogens and to determine their composition, and then quarantined to ensure they were not obtained during the seroconversion period of an infectious disease. Aliquots of every stool preparation are kept for future reference in case of an adverse event. Even in such a best-practice scenario, microorganisms considered innocuous might be harmful in certain contexts, such as immunocompromised or severely ill patients (who would be likelier candidates for FMT), and in-patient transmission of resistance genes could occur even from stool lacking detectable pathogens. It might thus be worth monitoring higher-risk genetic elements, such as extended spectrum $\beta$-lactamase plasmids.

Despite being an ancient practice, our understanding of the mechanistic basis of successful FMT is still in its infancy. Attention to clinical trial design is essential to maximize what we can learn about when, why and how FMT is successful. This likely includes a precise characterization of the participating subjects and their microbiota, as well as that of the stool used, extensive longitudinal sampling and multi-layered analysis of such samples. The lessons learned from these trials can bring us closer to the ultimate goal of replacing FMT with transplantation of specific microbial consortia or even microbial products, thus enabling scalability and eliminating many of the risks associated with FMT.

Published online: 23 July 2019

https://doi.org/10.1038/s41564-019-0535-1 